\title{
THERMAL STRESS IN ANISOTROPIC CYLINDERS
}

\author{
by G. EASON \\ (Received 19th June, 1962)
}

\section{Introduction}

In recent years considerable attention has been given to problems of thermal stress in isotropic materials. Much of this work has been devoted to statical problems although there has been some work on problems with time dependence, for example the quasi-static solutions obtained by Sternberg (1) and Eason and Sneddon (2). A good deal of interest has also been shown in statical thermal stress problems when the material is anisotropic. For the type of material considered here statical problems have been investigated by Grechushnikov and Brodovskii (3) and Sirotin (4) among others. Little attention has been given, however, to time dependent thermal stress problems when the material is anisotropic.

The problem considered here is that of the stress in a cylinder resulting from an applied surface temperature, when the material of the cylinder has transverse curvilinear isotropy. It is assumed that the temperature and the resulting stresses depend on the radial co-ordinate and the time only. A general solution of the basic equations is obtained using the Laplace transform. The particular problem of uniform surface temperature suddenly applied to a solid cylinder is then considered. The cylinder is assumed to have a stress free surface and to be initially at constant temperature. Inertia effects are neglected throughout so that the treatment is quasi-static and the solution obtained is unlikely to be valid immediately after the temperature is applied.

\section{The Basic Equations}

Let $r, \theta, z$ be cylindrical polar co-ordinates. In the problem considered here all quantities are assumed to depend on the radial co-ordinate $r$ and the time $t$ only. Under these conditions the heat conduction equation is

$$
\frac{\partial^{2} T}{\partial r^{2}}+\frac{1}{r} \frac{\partial T}{\partial r}=\frac{1}{\kappa} \frac{\partial T}{\partial t},
$$

where $T$ is the temperature of the body, $\kappa$ is the diffusivity and the coupling term has been neglected. For material with transverse isotropy about the radius vector the stress-strain relations, under plane strain conditions, are

$$
\sigma_{r}=c_{11} \frac{\partial u}{\partial r}+c_{12} \frac{u}{r}-\lambda_{1} T
$$




$$
\sigma_{\theta}=c_{12} \frac{\partial u}{\partial r}+c_{22} \frac{u}{r}-\lambda_{2} T,
$$

where $u$ is the radial displacement, $\sigma_{r}$ and $\sigma_{\theta}$ are the radial and circumferential stress components respectively, $c_{11}, c_{12}, c_{22}$ are the elastic constants of the material and $\lambda_{1}, \lambda_{2}$ are the stress-temperature coefficients (Hearman (5) p. 66). The remaining equation is the equation of equilibrium

$$
\frac{\partial \sigma_{r}}{\partial r}+\frac{1}{r}\left(\sigma_{r}-\sigma_{\theta}\right)=0
$$

The neglect of the coupling term in (1) and of the inertia term in (4) means that the discussion is essentially quasi-static and that the results obtained are unlikely to be valid immediately after any disturbance of the temperature distribution.

It is convenient to introduce dimensionless space and time co-ordinates defined by

$$
R=r / a, \quad \tau=\kappa t / a^{2},
$$

where $a$ is some typical length of the body. Equations (1)-(4) now become

$$
\begin{array}{r}
\frac{\partial^{2} T}{\partial R^{2}}+\frac{1}{R} \frac{\partial T}{\partial R}=\frac{\partial T}{\partial \tau}, \ldots \ldots . . \\
a \sigma_{r}=c_{11} \frac{\partial u}{\partial R}+c_{12} \frac{u}{R}-a \lambda_{1} T, \\
a \sigma_{\theta}=c_{12} \frac{\partial u}{\partial R}+c_{22} \frac{u}{R}-a \lambda_{2} T, \\
\frac{\partial \sigma_{r}}{\partial R}+\frac{1}{R}\left(\sigma_{r}-\sigma_{\theta}\right)=0 . \ldots .
\end{array}
$$

Equations (6)-(9) are the basic equations and their solution will now be considered.

\section{Solution of the Basic Equations}

To obtain a solution of equations (6)-(9) we introduce the Laplace transform $\bar{f}$ of the function $f$ defined by

$$
f(R, s)=\int_{0}^{\infty} f(R, \tau) e^{-s \tau} d \tau
$$

If each of equations (6)-(9) is multiplied by $\exp (-s \tau)$ and the resulting equations are integrated from 0 to $\infty$ with respect to $\tau$ it is found that

$$
\frac{d^{2} \bar{T}}{d R^{2}}+\frac{1}{R} \frac{d \bar{T}}{d R}=s \bar{T}
$$




$$
\begin{gathered}
a \bar{\sigma}_{r}=c_{11} \frac{d \bar{u}}{d R}+c_{12} \frac{\bar{u}}{R}-a \lambda_{1} \bar{T}, . \\
a \bar{\sigma}_{\theta}=c_{12} \frac{d \bar{u}}{d R}+c_{22} \frac{\bar{u}}{R}-a \lambda_{2} \bar{T}, \\
\frac{d \bar{\sigma}_{r}}{d R}+\frac{1}{R}\left(\bar{\sigma}_{r}-\bar{\sigma}_{\theta}\right)=0, \ldots .
\end{gathered}
$$

where it has been assumed that the body is at zero temperature when $\tau=0$.

Equation (11) has the solution

$$
\bar{T}=A I_{0}(R \sqrt{ } s)+B K_{0}(R \sqrt{ } s),
$$

where $A, B$ are constants and $I_{0}(R \sqrt{ } s)$ and $K_{0}(R \sqrt{ } s)$ are Bessel functions of imaginary argument of zero order and of the first and second kinds respectively. Substituting from (12) and (13) into (14) yields the equation

$$
\frac{d^{2} \bar{u}}{d R^{2}}+\frac{1}{R} \frac{d \bar{u}}{d R}-v^{2} \frac{\bar{u}}{R^{2}}=\alpha_{1} \frac{d \bar{T}}{d R}+\left(\alpha_{1}-\alpha_{2}\right) \frac{\bar{T}}{R}
$$

where

$$
v=\left(c_{22} / c_{11}\right)^{\frac{1}{2}}, \quad \alpha_{1}=a \lambda_{1} / c_{11}, \quad \alpha_{2}=a \lambda_{2} / c_{11}
$$

The method of variation of parameters gives as a solution of (16)

$$
\bar{u}=R^{v}\{C-\bar{\psi}(R)\}+R^{-v}\{D+\bar{\phi}(R)\} .
$$

where the functions $\bar{\phi}(R), \bar{\psi}(R)$ are defined by

$$
\begin{aligned}
\bar{\phi}(R) & =\frac{1}{2 v} \int_{R}^{1}\left\{\alpha_{1} R \frac{d \bar{T}}{d R}+\left(\alpha_{1}-\alpha_{2}\right) \bar{T}\right\} R^{v} d R \\
& =\frac{1}{2 v}\left\{\alpha_{1}\left(\bar{P}-\bar{T} R^{v+1}\right)-\left(v \alpha_{1}+\alpha_{2}\right) \int_{R}^{1} \bar{T} R^{v} d R\right\}, \ldots \\
\bar{\psi}(R) & =\frac{1}{2 v} \int_{R}^{1}\left\{\alpha_{1} R \frac{d \bar{T}}{d R}+\left(\alpha_{1}-\alpha_{2}\right) \bar{T}\right\} R^{-v} d R \\
& =\frac{1}{2 v}\left\{\alpha_{1}\left(\bar{P}-\bar{T} R^{1-v}\right)+\left(v \alpha_{1}-\alpha_{2}\right) \int_{R}^{1} \bar{T} R^{-v} d R\right\},
\end{aligned}
$$

and $P$ is the value of $T$ when $R=1$. The functions $\phi(R), \Psi(R)$ are to be regarded as the Laplace transforms of functions $\phi(R)$ and $\psi(R)$ respectively. Equations (12) and (13) give expressions for the transformed stress components of the forms

$$
\begin{aligned}
& a \bar{\sigma}_{r}=\left(v c_{11}+c_{12}\right) R^{v-1}\{C-\bar{\psi}(R)\}-\left(v c_{11}-c_{12}\right) R^{-(v+1)}\{D+\bar{\phi}(R)\}-c_{11} \alpha_{1} \bar{T}, \\
& a \bar{\sigma}_{\theta}=\left(c_{22}+v c_{12}\right) R^{v-1}\{C-\bar{\psi}(R)\}+\left(c_{22}-v c_{12}\right) R^{-(v+1)}\{D+\bar{\phi}(R)\}-c_{11} \alpha_{2} \bar{T}
\end{aligned}
$$

The solutions of the basic equations obtained here are quite general and may be used as the basis for obtaining solutions to problems such as that of a 
cylindrical tube with prescribed surface temperature as well as the cylindrical rod considered here.

\section{The Solid Cylinder}

As an example of the application of the basic solutions obtained in the previous section the problem of a solid cylinder will be considered. It is assumed that the surface of the cylinder is stress free and that the applied temperature has the constant value $Q$ for $t>0$. Let the cylinder have radius $a$. The conditions to be applied when $R=1$ are therefore

$$
\begin{aligned}
& T=P= \begin{cases}0 & t<0, \\
Q & t>0,\end{cases} \\
& \bar{\sigma}_{\mathrm{r}}=0,
\end{aligned}
$$

so that taking the Laplace transforms we find

$$
\begin{aligned}
\bar{T}=\bar{P} & =Q / s, \\
\sigma_{r} & =0, \ldots
\end{aligned}
$$

when $R=1$. The further conditions to be applied are that $T$ and $u$ remain finite as $r \rightarrow 0$, so that $\bar{T}$ and $\bar{u}$ must be finite as $R \rightarrow 0$. Inserting these conditions in (15), (18) and (21) results in

$$
\begin{aligned}
& B=0 \text {, } \\
& D=-\bar{\phi}(0) \text {, } \\
& A=\frac{Q}{s I_{0}(\sqrt{ } s)}, \\
& \beta C=c_{11} \alpha_{1} Q / s-\left(v c_{11}-c_{12}\right) \phi(0),
\end{aligned}
$$

where

$$
\beta=v c_{11}+c_{12} \text {. }
$$

The expressions for the transformed temperature and displacement are now obtained in the forms

$$
\begin{array}{r}
\bar{T}=\frac{Q I_{0}(R \sqrt{ } s)}{s I_{0}(\sqrt{ } s)}, \ldots \ldots \ldots \ldots \ldots \ldots \ldots \ldots \ldots \ldots \ldots \\
\bar{u}=R^{v}\left\{c_{11} \alpha_{1} Q / s-\left(v c_{11}-c_{12}\right) \phi(0)-\beta \bar{\psi}(R)\right\} / \beta+R^{-v}\{\phi(R)-\bar{\phi}(0)\} .
\end{array}
$$

The inversion theorem for the Laplace transform now gives

$$
\begin{array}{r}
T=\frac{Q}{2 \pi i} \int_{y-i \infty}^{\gamma+i \infty} \frac{I_{0}(R \sqrt{ } s) e^{s \tau} d s}{s I_{0}(\sqrt{ } s)} \ldots \ldots \ldots \ldots \ldots \ldots \ldots \ldots \ldots \\
u=R^{v}\left\{c_{11} \alpha_{1} Q-\left(v c_{11}-c_{12}\right) \phi(0)-\beta \psi(R)\right\} / \beta+R^{-v}\{\phi(R)-\phi(0)\},
\end{array}
$$

where $\gamma$ is a real constant chosen so that all singularities of the integrand of (32) 
lie to the left of the line $s=\gamma$ in the complex s-plane, and $\phi(R), \psi(R)$ are defined by

$$
\begin{aligned}
& \phi(R)=\left\{\alpha_{1}\left(Q-T R^{v+1}\right)-\left(v \alpha_{1}+\alpha_{2}\right) \int_{R}^{1} T R^{v} d R\right\} / 2 v, \\
& \psi(R)=\left\{\alpha_{1}\left(Q-T R^{1-v}\right)+\left(v \alpha_{1}-\alpha_{2}\right) \int_{R}^{1} T R^{-v} d R\right\} / 2 v .
\end{aligned}
$$

Equations (21) and (22) lead to expressions for the stress components of the forms

$$
\begin{aligned}
\alpha \sigma_{r}=-c_{11} \alpha_{1} T & -\left(v c_{11}-c_{12}\right) R^{-(v+1)}\{\phi(R)-\phi(0)\} \\
& +R^{v-1}\left\{c_{11} \alpha_{1} Q-\left(v c_{11}-c_{12}\right) \phi(0)-\beta \psi(R)\right\}, \quad \ldots \ldots \ldots \ldots . . . . . . . . \\
\alpha \sigma_{\theta}=-c_{11} \alpha_{2} T & +\left(c_{22}-v c_{12}\right) R^{-(v+1)}\{\phi(R)-\phi(0)\} \\
& +\left(v c_{12}+c_{22}\right) R^{v-1}\left\{c_{11} \alpha_{1} Q-\left(v c_{11}-c_{12}\right) \phi(0)-\beta \psi(R)\right\} / \beta
\end{aligned}
$$

It is seen from equations (32)-(37) that once $T$ is determined all other quantities may be found by evaluating the single integrals occurring in (34) and (35). The integral for $T$ is known (see Carslaw and Jaeger (6) p. 328). It has poles at $s=0$ and $s=-\lambda_{n}^{2}$ where $\lambda_{n}$ is the $n$th positive root of the equation

$$
J_{0}(\lambda)=0 \text {, }
$$

where $J_{v}(\lambda)$ is a Bessel function of the first kind of order $v$. It is now found that

$$
\begin{aligned}
& T=Q\left\{1-2 \sum_{n=1}^{\infty} \frac{J_{0}\left(R \lambda_{n}\right) e^{-\lambda_{n}^{2} \tau}}{\lambda_{n} J_{1}\left(\lambda_{n}\right)}\right\} \\
& \phi(R)=\frac{Q}{2 v}\left\{\frac{\left(\alpha_{1}-\alpha_{2}\right)}{1+v}\left(1-R^{1+v}\right)+2 \alpha_{1} R^{1+v} \sum_{n=1}^{\infty} \frac{J_{0}\left(R \lambda_{n}\right) e^{-\lambda_{n}^{2} z}}{\lambda_{n} J_{1}\left(\lambda_{n}\right)}\right. \\
& \left.+2\left(v \alpha_{1}+\alpha_{2}\right) \sum_{n=1}^{\infty} \frac{e^{-\lambda_{n}^{2} \tau}}{\lambda_{n} J_{1}\left(\lambda_{n}\right)} \int_{R}^{1} R^{v} J_{0}\left(R \lambda_{n}\right) d R\right\}, \\
& \psi(R)=\frac{Q}{2 v}\left\{\frac{\left(\alpha_{1}-\alpha_{2}\right)}{v-1}\left[R^{1-v}-1\right]+2 \alpha_{1} R^{1-v} \sum_{n=1}^{\infty} \frac{J_{0}\left(R \lambda_{n}\right) e^{-\lambda_{n}^{2} \tau}}{\lambda_{n} J_{1}\left(\lambda_{n}\right)}\right. \\
& \left.-2\left(v \alpha_{1}-\alpha_{2}\right) \sum_{n=1}^{\infty} \frac{e^{-\lambda_{n}^{2} \tau}}{\lambda_{n} J_{1}\left(\lambda_{n}\right)} \int_{R}^{1} R^{-v} J_{0}\left(R \lambda_{n}\right) d R\right\}, \quad v \neq 1, \\
& \psi(R)=\frac{1}{2} Q\left\{-\left(\alpha_{1}-\alpha_{2}\right) \ln R+2 \alpha_{1} \sum_{n=1}^{\infty} \frac{J_{0}\left(R \lambda_{n}\right) e^{-\lambda_{n}^{2} \tau}}{\lambda_{n} J_{1}\left(\lambda_{n}\right)}\right. \\
& \left.-2\left(\alpha_{1}-\alpha_{2}\right) \sum_{n=1}^{\infty} \frac{e^{-\lambda_{n}^{2} \tau}}{\lambda_{n} J_{1}\left(\lambda_{n}\right)} \int_{R}^{1} \frac{1}{R} J_{0}\left(R \lambda_{n}\right) d R\right\}, \quad v=1 .
\end{aligned}
$$

Equations (39)-(42) give a solution to our problem, the displacement and stress components are found by substituting into (33), (36) and (37). The integrals 
occurring in (40) may be evaluated explicitly when $v$ is an odd integer. Due to the way in which $\phi(R)$ and $\psi(R)$ were defined in (19) and (20) it is seen that

$$
\phi(1)=\psi(1)=0,
$$

so that the surface displacement and stress depends on $\phi(0)$ alone.

The results obtained in this section will now be specialised to the case of an isotropic solid.

\section{The Isotropic Solid}

The particular results for an isotropic solid are obtained by writing

$$
\alpha_{1}=\alpha_{2}=\alpha, \quad c_{11}=c_{22}, \quad v=1 .
$$

Equations (40) and (42) now give

$$
\begin{gathered}
\phi(R)=\alpha Q \sum_{n=1}^{\infty} \frac{e^{-\lambda_{n}^{2} \tau}}{\lambda_{n}^{2} J_{1}\left(\lambda_{n}\right)}\left\{R^{2} \lambda_{n} J_{0}\left(R \lambda_{n}\right)\right. \\
\left.\quad+2 J_{1}\left(\lambda_{n}\right)-2 R J_{1}\left(\lambda_{n}\right)\right\}, \\
\psi(R)=\alpha Q \sum_{n=1}^{\infty} \frac{e^{-\lambda_{n}^{2} \tau} J_{0}\left(R \lambda_{n}\right)}{\lambda_{n} J_{1}\left(\lambda_{n}\right)},
\end{gathered}
$$

as the basic results from which to determine the displacement and stress components.

\section{REFERENCES}

(1) E. Sternaerg, Proc. Koninkl. Nederl. Akad. Wet., Ser. B 60 (1957), 396.

(2) G. Eason and I. N. SNeddon, Proc. Roy. Soc. Edin., A 65 (1959), 143.

(3) B. N. GREChUSHNIKov and D. BrodovskII, Kristallografiya, 1 (1956), 597.

(4) Y. I. SIROTIN, Kristallografiya, 1 (1956), 708.

(5) R. F. S. Hearman, An introduction to applied anisotropic elasticity (Oxford University Press, 1961).

(6) H. S. Carslaw and J. C. JAEger, Conduction of heat in solids, 2nd edn. (Oxford University Press, 1959).

Department of Mathematics

Royal College of Science and Technology

Glasgow, C.1 